\title{
Neighbourhood built environment associations with body size in adults: mediating effects of activity and sedentariness in a cross-sectional study of New Zealand adults
}

\author{
Melody Oliver ${ }^{1 *}$, Karen Witten ${ }^{2}$, Tony Blakely ${ }^{3}$, Karl Parker $^{2}$, Hannah Badland ${ }^{4}$, Grant Schofield $^{1}$, Vivienne Ivory ${ }^{3}$,
} Jamie Pearce ${ }^{5}$, Suzanne Mavoa ${ }^{2,4}$, Erica Hinckson ${ }^{1}$, Paul Sweetsur ${ }^{2}$ and Robin Kearns ${ }^{6}$

\begin{abstract}
Background: The aim of this study was to determine the associations between body size and built environment walkability variables, as well as the mediating role of physical activity and sedentary behaviours with body size.

Methods: Objective environment, body size (body mass index (BMI), waist circumference (WC)), and sedentary time and physical activity data were collected from a random selection of 2033 adults aged 20-65 years living in 48 neighbourhoods across four New Zealand cities. Multilevel regression models were calculated for each comparison between body size outcome and built environment exposure.
\end{abstract}

Results and Discussion: Street connectivity and neighborhood destination accessibility were significant predictors of body size (1 SDchange predicted a 1.27 to $1.41 \%$ reduction in BMI and a 1.76 to $2.29 \%$ reduction in WC). Significantrelationships were also observed for streetscape (1 SD change predicted a $1.33 \%$ reduction in BMI) anddwelling density (1 SD change predicted a $1.97 \%$ reduction in BMI). Mediation analyses revealed asignificant mediating effect of physical activity on the relationships between body size and street connectivity and neighbourhood destination accessibility (explaining between 10.4 and $14.6 \%$ of the total effect). No significant mediating effect of sedentary behaviour was found. Findings from this cross-sectional study of a random selection of New Zealand adults are consistent with international research. Findings are limited to individual environment features only; conclusions cannot be drawn about the cumulative and combined effect of individual features on outcomes.

Conclusions: Built environment features were associated with body size in the expected directions. Objectively-assessed physical activity mediated observed built environment-body size relationships.

Keywords: Obesity, Epidemiology, Physical activity, Geographic information systems, Body mass index, Urban design, Walkability, New Zealand

\section{Background}

The global obesity epidemic poses one of the greatest challenges in modern public health [1]. Contributors to obesity are mutlifaceted and complex, with behavioural (nutrition, physical activity), metabolic, and environmental factors implicated [2,3]. Increased understanding of factors associated with obesity at the population level is

\footnotetext{
* Correspondence: melody.oliver@aut.ac.nz

'Human Potential Centre, Auckland University of Technology, Auckland, New Zealand

Full list of author information is available at the end of the article
}

fundamental to efficacious intervention development and implementation.

Escalating interest in the role of built environments (BEs) as being obesogenic, or obesity promoting, has been demonstrated in the last decade $[1,4,5]$. Emerging evidence suggests relationships between obesity and various $\mathrm{BE}$ attributes in adults [2, 4, 6-11]; however, to what degree the $\mathrm{BE}$ is associated with obesity, and the mechanisms by which this occurs, are yet to be fully determined [2]. Theories suggest direct pathways between the $\mathrm{BE}$ and dietary and physical activity behaviours, with

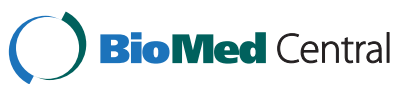

(c) 2015 Oliver et al. Open Access This article is distributed under the terms of the Creative Commons Attribution 4.0 International License (http://creativecommons.org/licenses/by/4.0/), which permits unrestricted use, distribution, and reproduction in any medium, provided you give appropriate credit to the original author(s) and the source, provide a link to the Creative Commons license, and indicate if changes were made. The Creative Commons Public Domain Dedication waiver (http://creativecommons.org/publicdomain/zero/1.0/) applies to the data made available in this article, unless otherwise stated. 
subsequent impacts on body size (concurrently acknowledging individual and social environment factors that may influence these relationships) [4, 12, 13]. Stronger and more consistent relationships have been found between $\mathrm{BE}$ characteristics and physical activity than for food environments $[14,15]$. For example, cross-sectional (predominantly US) research has shown clear relationships between $\mathrm{BE}$ features and physical activity behaviours (particularly walking) in adults $[8,16,17]$.

The magnitude of the associations between environmental features and physical activity can be considerable - earlier work in the Understanding the Relationship Between Activity and Neighbourhoods (URBAN) study in New Zealand demonstrated moderate populationwide effects of $B E$ features with self-reported and objectively-assessed physical activity in adults [18]. A one standard deviation (SD) higher destination access, street connectivity, and dwelling density was associated with increased odds of any self-reported physical activity via active transport, walking, or leisure-time activities (ranges in effect sizes from 21 to $44 \%$ ), and $7 \%$ higher objectivelyassessed (via accelerometer) physical activity levels.

Investigating these relationships poses a number of specific challenges, including inconsistencies in conceptual underpinnings, delimitation of geographical boundaries, operationalization of neighbourhoods (e.g., residential and non-residential spaces), and measures of $\mathrm{BE}$ features $[2,4,10]$ across studies. Within-study homogeneity in demography and $\mathrm{BE}$ attributes has further limited our understanding of these relationships $[4,19]$. It is also not clear how much of a contribution preferences for living in more walkable neighbourhoods and 'self-selection' into preferred neighbourhoods may have in explaining the observed association of $\mathrm{BE}$ with physical activity and body size; the extent of such confounding bias is not well quantified or understood in existing research $[7,9,13,20-22]$. The likely collinearity of $\mathrm{BE}$ features also makes it difficult to isolate the effects of individual $\mathrm{BE}$ attributes on outcomes of interest. With regard to the behaviours and outcomes, a majority of studies have relied on participants' selfreported physical activity [23] and height and weight (to calculate the dependent variable, BMI) [19, 23, 24] respectively; measures known to be limited by social desirability bias $[25,26]$. BMI has been consistently employed as the dependent outcome. However, this measure is only a proxy for body fatness and differential health risks may be found across individuals of differing ethnicities. Furthermore, alternative proxy measures - WC in particular - have been shown to have stronger associations than BMI with health status and chronic conditions in adults [27].

It is highly likely that physical activity mediates any association between $\mathrm{BE}$ features and body size, but few studies have considered this relationship [7, 23, 28-32]. Such examinations are important to test the theoretical coherence of the association between the $\mathrm{BE}$ and body size; that is, if physical activity does not contribute somewhat to this relationship, it is possible that systematic error, most notably confounding, may exist. Furthermore, little is known of the potential mediating effect of sedentary behaviour on this relationship. A clear pathway exists between sedentary time (especially prolonged sitting) and body size [33] and evidence suggests a link between the $\mathrm{BE}$ and sedentary time (particularly via sitting during motorised transport) [34]. Thus it is possible that any observed relationship between $\mathrm{BE}$ features and body size may be mediated by sedentary time.

In this paper we extend on earlier research through analysis of objective measures of body size (BMI, WC) and physical activity and sedentary behaviour (accelerometry) collected from a random selection of participants living within urban neighbourhoods in New Zealand, purposively selected to maximise area-level variability in walkability. Building on our earlier investigations in the URBAN Study [18], we examine the effect of objectively assessed physical activity and sedentary behaviours on the association between commonly-assessed $\mathrm{BE}$ features conceptualised as being associated with physical activity and body size. Hypotheses were that body size would be negatively associated with individual $\mathrm{BE}$ features under investigation, and that objectively-assessed physical activity (by decreasing body size) and sedentary behaviours (by increasing body size) would mediate this relationship.

\section{Methods}

Data were drawn from the URBAN Study, for which the methods have been previously reported [35]. This crosssectional observational study was conducted in four New Zealand cities (Waitakere and North Shore in the Auckland region, Christchurch, and Wellington) between April 2008 and September 2010 (total population $=920,000)$. The URBAN Study is the New Zealand arm of the multi-country International Physical Activity and Environment Network (IPEN); involving replicate protocols across 12 member countries for assessment of dependent and independent variables [36]. Ethical approval to conduct the research was granted by the host institutions' ethics committees (AUTEC 07/126; MUHECN 07/045). All participants provided informed written consent to participate.

\section{Neighbourhood and participant selection}

A walkability index was calculated for every meshblock (the smallest geographic census area unit in New Zealand; approximating to 100 people) across the four cities $(n=7,614)$. The index was calculated using geographic 
information systems (GIS)-derived spatial measures of dwelling density, land-use mix, street connectivity, and retail floor area ratio as described elsewhere [18, 37]. Summary scores (average of the mesh-block level walkability z-score values) were calculated for each neighbourhood and neighbourhoods were partitioned into walkability tertiles (low/medium/high). Meshblocks in the highest and lowest tertiles of derived walkability scores were deemed eligible for selection.

Distribution of usual Māori residents domiciled within each mesh-block within the four cities was estimated by using 2006 census data [38]. Following the walkability index procedures, the mesh-block Māori population density was classified into deciles and recoded into values from 1 (1st decile) to 10 (10th decile) for each city. Māori comprise $14.6 \%$ of the resident population. They are the second largest ethnic group (after New Zealand Pākehā/ European) in New Zealand [39].

Study neighbourhoods were defined as clusters of five contiguous meshblocks within the same strata (i.e., high or low walkability, and high or low Māori population). This was a deliberate approach to ensure geographical spread and facilitate ethnic diversity within each region. Overall, 48 neighbourhoods were selected (12 per city). Random start points were generated within each neighbourhood, and trained interviewers then approached every $\mathrm{n}^{\text {th }}$ house (determined by neighbourhood dwelling density) following a designated walk path for household enumeration and participant invitation to the study. A maximum of five visits were made to each eligible household. One adult per household was invited to participate, using next-birthday enumeration. Participant exclusion criteria were: outside the 20 to 65 year age range, not living in the household during the measurement period or for the 3 months prior, unable to speak English, or being walking-impaired.

\section{Protocol}

Upon participant agreement, two home visits were scheduled. At the first home visit, informed consent was gained and participants were provided with an accelerometer to wear during waking hours over the next seven consecutive days (excluding water-based activities) alongside verbal and written instructions for accelerometer wear. Accelerometers were collected at the second home visit (approximately eight days later), at which time body size measures were taken and the study survey was administered by the interviewer.

\section{Measures}

Body size: Height $(\mathrm{m})$ to the nearest $0.1 \mathrm{~cm}$ and weight $(\mathrm{kg})$ to the nearest $0.1 \mathrm{~kg}$ were assessed using a stadiometer and calibrated Seca scales, respectively. BMI was calculated as weight $(\mathrm{kg}) /$ height $\left(\mathrm{m}^{2}\right)$. Waist circumference was measured at the mid-point between the iliac crest and the lateral costal margin to the nearest $0.1 \mathrm{~cm}$ using a Lufkin W606PM tape. International Society for the Advancement of Kinanthropometry protocols were followed for all body size measurements [40].

Built environment: GIS-derived spatial measures of the $\mathrm{BE}$ were generated as follows: (1) Dwelling density, number of dwellings/residential land area in a meshblock (per square kilometre); (2) Street connectivity, number of intersections with 3 or more intersecting streets per square kilometres within a meshblock; (3) Land use mix was calculated using the entropy index formula of Leslie et al. [41] (where $\mathrm{k}$ is the category of land use (residential, commercial/retail, public open space, industrial, other), $\mathrm{p}$ is the proportion of the land area in the specific land use (where the denominator is the total non-water/non-road area), and $\mathrm{N}$ is the number of land use categories), and (4) the 'Neighbourhood Destination Accessiblity Index', which is a measure of the intensity of neighbourhood destination opportunities within an $800 \mathrm{~m}$ buffer of the meshblock centroid [42]. Areas in water and roads were excluded from the area-denominator in all calculations. Neighbourhood level social deprivation was derived from the 2006 Census, providing area-level socioeconomic information at the meshblock level [43].

Streetscape: The Systematic Pedestrian and Cycling Environment Scan (SPACES) has demonstrated reliability for measuring environmental supports or barriers (e.g., safety, aesthetics) for walking and cycling [44]. This audit tool was modified for use in the New Zealand context [45] and implemented by trained fieldworkers. A total of 12 street segments were selected sequentially from a random start point in each neighbourhood. Fieldworkers audited each street segment (total $n=576$ ) and individual street segment scores were combined for each neighbourhood.

Physical activity: Objective measurement of physical activity was captured using an Actical accelerometer worn above the right iliac crest on an elasticated belt (Mini-Mitter, Sunriver, OR). Units were set to collect data in $30 \mathrm{~s}$ epochs. At the end of the measurement period, data were downloaded within the manufacturer software, and raw accelerometer data were extracted from the Actical Export File (Version 02.10) listings for each participant. The raw data were read and plotted in SAS (version 9.2; SAS Institute, Cary, NC) for checking. Research officers were instructed to use event markers to record device delivery and collection so participants' physical activity data were easily identified and extracted. Physical activity printouts were checked for excessive accelerometer counts (approximating $>1000$ counts per minute) and if identified, the accelerometer was considered faulty and that participants' data removed from 
analyses $(n=26)$. Consequently, that day's data and all successive data were set to missing. Data from participants engaged in shift work $(n=40)$ were also removed because timing of their daily activities and sleep varied from day to day. A conservative non-wear criterion of 60 consecutive minutes of 0 counts was employed [46] and these data excluded from analyses. Thereafter, any periods of less than 60 consecutive minutes during which the accelerometer was worn were also set to missing and these data excluded from analyses. This approach was employed to reduce the possibility of including data collected from movement of the accelerometer when not worn, or in case participants wore the accelerometer only while exercising. Cleaned data were reduced to mean number of accelerometer counts per hour while worn during weekend days and weekdays (weighted by individual wear-time). The count threshold for sedentary time was $<100$ counts per minute [47].

Demographic information: The interviewer-administered survey captured information on respondents' date of birth (to calculate age in years), sex, educational qualifications, marital status, employment status, household income, and car access. Combined household income before tax for the previous 12 months (in New Zealand Dollars, NZD) was assessed using seven categories; zero, $<\$ 20,000, \$ 20,001$ to $\$ 40,000, \$ 40,001$ to $\$ 60,000, \$ 60,001$ to $\$ 80,000, \$ 80,001$ to $\$ 100,000$, and $>\$ 100,000$. Data were later aggregated to five categories reflecting bands of $\$ 20,000$; from $<\$ 20,000$ to $>\$ 100,000$ per annum. The median annual household income for New Zealand in 2010 was $\$ 75,700$ [48].

Neighbourhood preference: Preference for a more suburban (less walkable) or urban (more walkable) neighbourhood (assuming housing cost, quality of schools, and mix of people were constant across neighbourhood type) was determined using survey items developed by Levine et al. [49], for which face validity has been established.

\section{Analyses}

Significant correlations were observed across neighbourhood characteristics (Table 1); therefore each neighbourhood exposure was modeled individually with the two body size outcome measures (BMI, WC). To facilitate comparability across exposures, BE features were rescaled by dividing each feature by their SD calculated across all neighbourhoods.

Accelerometer counts per hour, BMI, and WC were logtransformed to achieve approximately normal distributions. Non-linear relationships for the explanatory variables were investigated but none were found. Table 2 shows the correlations between outcome measures and neighbourhood level deprivation. Evidence suggests that physical activity and sedentary behaviours have distinct and independent effects on health $[50,51]$; accordingly, these variables were considered in separate models as indicated below.

Multi-level regression analyses were conducted in $\mathrm{R}$ using the package "lme4" [52] with random effects to account for the clustering of individuals within neighbourhoods $(n=48)$. Separate models were run to predict the associations between each BE measure with (a) BMI and (b) WC using three approaches as follows: Modelling approach 1 (hereafter termed Model 1 for simplicity) adjusted for sex, age, ethnicity, individual level socioeconomic factors (marital status, education, income, employment, car access), neighbourhood-level deprivation (theorized as a confounder and due to observed correlations with BE features and with body size - discussed in Results below), and neighbourhood preference (i.e., model fully adjusted for theorized and measured confounders to estimate total effect of BE on BMI); Approach 2 (hereafter termed Model 2) additionally included physical activity as a potential mediator (using the log of accelerometer counts per hour); and Approach 3 (hereafter termed Model 3) was identical to Model 2, with physical activity replaced by percentage of time spent in sedentary behaviour. Outcome and mediating models were created for the above models and mediation analysis was then performed using the "mediation" package in $\mathrm{R}[53,54]$. This was used to estimate the indirect effects between the independent variable (i.e., the $\mathrm{BE}$ variables) and body size (i.e., BMI and $\mathrm{WC}$ ) via the mediating variables (i.e., physical activity and percentage of time spent in sedentary behaviour). A quasi-Bayesian approximation was used to calculate the $p$-value using 5000 Monte Carlo draws.

Table 1 Correlation matrix showing associations between neighbourhood-level characteristics ( $n=48$ neighbourhoods)

\begin{tabular}{|c|c|c|c|c|c|c|}
\hline & Dwelling density & Street connectivity & Mixed land use & $\mathrm{NDAl}$ & Streetscape & NZDep06 \\
\hline Dwelling density & 1.00 & & & & & \\
\hline Street connectivity & $0.89^{*}$ & 1.00 & & & & \\
\hline Mixed land use & -0.02 & $0.09^{*}$ & 1.00 & & & \\
\hline $\mathrm{NDAl}$ & $0.71^{*}$ & $0.75^{*}$ & $0.10^{*}$ & 1.00 & & \\
\hline Streetscape & $0.19^{*}$ & $0.26^{*}$ & $0.34^{*}$ & $0.39^{*}$ & 1.00 & \\
\hline NZDep06 & $0.36^{*}$ & $0.42^{*}$ & 0 & $0.38^{*}$ & 0.02 & 1.00 \\
\hline
\end{tabular}


Table 2 Correlation matrix showing associations between outcome measures and neighbourhood-level deprivation $(n=48$ neighbourhoods)

\begin{tabular}{|c|c|c|c|c|c|}
\hline & $\begin{array}{l}\text { Log-transformed accelerometer } \\
\text { counts per hour }\end{array}$ & $\begin{array}{l}\text { Percentage of time in } \\
\text { Sedentary behaviour }\end{array}$ & $\begin{array}{l}\text { Log-transformed } \\
\text { waist circumference }\end{array}$ & Log-transformed BMI & NZDep06 \\
\hline $\begin{array}{l}\text { Log-transformed accelerometer } \\
\text { counts per hour }\end{array}$ & 1.00 & & & & \\
\hline $\begin{array}{l}\text { Percentage of time in } \\
\text { Sedentary behaviour }\end{array}$ & $-0.72^{*}$ & 1.00 & & & \\
\hline $\begin{array}{l}\text { Log-transformed waist } \\
\text { circumference }\end{array}$ & $-0.15^{*}$ & $0.13^{*}$ & 1.00 & & \\
\hline Log-transformed BMI & $-0.09^{*}$ & $0.10^{*}$ & $0.84^{*}$ & 1.00 & \\
\hline NZDep06 & -0.05 & 0.03 & $0.11^{*}$ & $0.15^{*}$ & 1.00 \\
\hline
\end{tabular}

Note: NZDep06 New Zealand Deprivation Index 2006

${ }^{*} p<0.01$

\section{Results}

In total, 2033 adults participated (provided data from non-faulty accelerometers) in the study, reflecting a $44 \%$ response rate. All data provided by the 2033 participants were included in the analysis to reduce bias introduced when decisions are made to include or exclude data [55]. Participants contributed a total of $16,869,826$ counts per hour, averaging to 9,177 counts per hour across 1-7 days of data. Descriptive information for participants, outcome measures, and neighbourhood characteristics are provided in Table 3. The population sampled were predominantly female $(57 \%)$ and had at least some access to a car (93\%), consistent with the overall New Zealand population (51 \% female [38], $92 \%$ access to a motor vehicle [56]). Compared with the New Zealand adult population, a greater proportion were employed (86 versus $66 \%$ ) [57] and married (64 versus $45 \%)$ [38]. A correlation matrix of $\mathrm{BE}$ features and neighbourhood-level deprivation is provided in Table 1. Statistically significant positive correlations were observed between neighbourhood-level deprivation and dwelling density, street connectivity, and destination accessibility $(r=0.36,0.42$, and 0.38 , respectively, all $p<0.01)$. All $\mathrm{BE}$ features were significantly correlated with each other $(r=0.09$ to $0.89, p<0.01$ ), with the exception of dwelling density and mixed land use.

Values for correlations between outcome measures and neighbourhood-level deprivation are provided in Table 2. Correlations were all in the expected direction, with both body size measures being significantly correlated with each other $(r=0.84, p<0.01)$, while a negative correlation was observed between physical activity and sedentary behaviour $(r=-0.72, p<0.01)$. Small but significant correlations were also observed between body size variables and sedentary behaviour (positive), neighbourhood-level deprivation (positive), and physical activity (negative).

The estimates for all associations with BMI and waist circumference for Models 1 to 3 are presented in Tables 4 and 5 , respectively. In the fully adjusted model (accounting for individual characteristics and neighbourhood-level deprivation; Model 1), street connectivity, NDAI, and streetscape were significant predictors of BMI (a 1 SD increase in each was associated with between 1.27 and $1.41 \%$ reductions in BMI, $p<0.05)$. For the same model, 1 SD increases in dwelling density, street connectivity, and NDAI were associated with reductions in waist circumference values of $1.97,1.76$ and $2.29 \%$, respectively $(p<0.05)$.

Adjusting for the potential mediation by physical activity and sedentary behaviour (Models 2 and 3, respectively) each compared to our 'best' total estimate of the BE-BMI association in Model 1 saw $13.2 \%$ (street connectivity adjusting for time spent sedentary) to $14.6 \%$ (NDAI adjusting for physical activity) of the BE-BMI association explained, and 10.4 \% (NDAI adjusting for physical activity) to $14.3 \%$ (street connectivity adjusting for physical activity) of the BE-WC association explained. An inconsistent mediation effect was observed for physical activity in the BMI-dwelling density relationship, that is, a nonsignificant association was found for the total effect, yet a significant mediating effect was found for physical activity. Therefore, no interpretation of results for this model have been provided [58].

\section{Discussion}

Significant negative associations between objectivelyassessed BE characteristics and body size were observed in the current study. After adjusting for individual characteristics, neighbourhood preference, and neighbourhoodlevel deprivation, street connectivity, and destination accessibility were associated with reduced BMI and waist circumference (ranging from -1.27 to $-2.29 \%$ for a 1 $\mathrm{SD}$ change in each $\mathrm{BE}$ variable). Higher quality streetscape, as assessed by the NZ-SPACES tool, was also associated with reduced BMI, but this relationship did not hold true for waist circumference. Dwelling density was associated with reduced WC $(-1.97 \%, p=0.004)$, 
Table 3 Participant and neighbourhood characteristics

\begin{tabular}{|c|c|c|c|}
\hline $\begin{array}{l}\text { Variable } \\
\end{array}$ & Total $^{a}$ & Number & Percent \\
\hline Age & 2020 & & \\
\hline $15-29$ & & 450 & 22.3 \\
\hline $30-44$ & & 782 & 38.7 \\
\hline $45-54$ & & 462 & 22.9 \\
\hline $55-65$ & & 326 & 16.1 \\
\hline Ethnicity & 2033 & & \\
\hline Māori & & 241 & 11.9 \\
\hline Non Māori & & 1792 & 88.1 \\
\hline Sex & 2032 & & \\
\hline Male & & 853 & 42.0 \\
\hline Female & & 1179 & 58.0 \\
\hline Qualification & 2025 & & \\
\hline No Qualification & & 546 & 27.0 \\
\hline School & & 234 & 11.6 \\
\hline Post School & & 474 & 23.4 \\
\hline Tertiary & & 771 & 38.1 \\
\hline Marital status & 2028 & & \\
\hline Never married & & 447 & 22.0 \\
\hline Married & & 1302 & 64.2 \\
\hline Previous married & & 279 & 13.8 \\
\hline Income (\$NZ) & 1821 & & \\
\hline$=<\$ 40,000$ & & 421 & 23.1 \\
\hline$\$ 40.001-60,000$ & & 316 & 17.3 \\
\hline$\$ 60,001-80,000$ & & 271 & 14.8 \\
\hline$\$ 80,001-100,00$ & & 276 & 15.1 \\
\hline$>\$ 100,000$ & & 541 & 29.6 \\
\hline Employment & 2030 & & \\
\hline Full time & & 1182 & 58.2 \\
\hline Part time/Non-standard work & & 542 & 26.7 \\
\hline Unpaid & & 306 & 15.1 \\
\hline Car access & 2032 & & \\
\hline Un restricted & & 1633 & 80.4 \\
\hline Restricted & & 241 & 11.9 \\
\hline No car access & & 158 & 7.8 \\
\hline Neighbourhood preference & 2006 & & \\
\hline Strongly prefer walkable & & 700 & 34.9 \\
\hline Moderately prefer walkable & & 333 & 16.6 \\
\hline Neutral & & 286 & 14.3 \\
\hline Moderately prefer less walkable & & 207 & 10.3 \\
\hline Strongly prefer less walkable & & 480 & 23.9 \\
\hline Neighbourhood deprivation (NZDep06) & 2033 & & \\
\hline \multirow[t]{2}{*}{ Least deprived quintile } & & 419 & 20.6 \\
\hline & & 380 & 18.7 \\
\hline
\end{tabular}

Table 3 Participant and neighbourhood characteristics (Continued)

\begin{tabular}{|c|c|c|c|}
\hline & & 419 & 20.6 \\
\hline & & 435 & 21.4 \\
\hline Most deprived quintile & & 380 & 18.7 \\
\hline Exposure and outcome variables & Total & Mean & SD \\
\hline \multicolumn{4}{|l|}{ Body size } \\
\hline BMI & 2007 & 27.04 & 5.68 \\
\hline Waist circumference & 1994 & 88.60 & 15.01 \\
\hline Accelerometer-derived measures & 1838 & & \\
\hline $\begin{array}{l}\text { Physical activity (accelerometer counts } \\
\text { per hour }{ }^{b} \text { ) }\end{array}$ & & 9177 & 4803 \\
\hline $\begin{array}{l}\text { Sedentariness (percentage of time } \\
\text { sedentary) }\end{array}$ & & 57.49 & 11.89 \\
\hline Neighbourhood exposures ${ }^{c}$ & 2033 & & \\
\hline Dwelling density & & 5.87 & 2.74 \\
\hline Street connectivity & & 5.42 & 2.39 \\
\hline Mixed land use & & 5.69 & 2.08 \\
\hline NDAl & & 11.74 & 4.93 \\
\hline Streetscape & & 87.57 & 11.26 \\
\hline
\end{tabular}

Note: $B M I$ body mass index $\left(\mathrm{kg} / \mathrm{m}^{2}\right), n$ number, NDAl Neighbourhood Destination Accessibility Index, NZDep06 New Zealand Deprivation Index 2006, $\$ N Z$ New Zealand dollars, $S D$ standard deviation

aData were missing as follows: Age, $n=13$; sex, $n=1$; qualifications, $n=8$; marital status, $n=5$; income, $n=212$; employment status, $n=3$; car access, $n=1$; waist circumference, $n=39$; BMI, $n=26$; accelerometer data, $n=195$

${ }^{b}$ Accelerometer units were counts per hour while worn (weighted by hours of data recorded)

'Means and standard deviations of neighbourhood exposures were calculated across study neighbourhoods

and this relationship neared significance for BMI $(-1.10 \%, p=0.061)$.

When considering the results for the fully adjusted model, the differences found in body size may appear small. However, if we compare settings at either end of the $\mathrm{BE}$ spectrum, the consequences of living in these neighbourhoods on body size outcomes could be important. For example, when comparing the $5^{\text {th }}$ and $95^{\text {th }}$ percentiles of dwelling density in the current study (data reported in Witten et al. [18]), a SD of 3.14 is found. If the current study findings are applied to compare these two environments, a 3.14 SD in dwelling density would equate to a difference of approximately $1 \mathrm{BMI}$ unit in an adult, or roughly $3-4 \mathrm{~kg}$, dependent on height. It is important to note that observed associations are not independent of each other and that the approach taken to analysis in the current study does not enable conclusions to be drawn about the cumulative and combined effect of individual $\mathrm{BE}$ features on outcomes. For instance, if we consider street connectivity and destination accessibility, a number of scenarios may exist - one could be an indicator of the other, the two factors may interact, or they could have completely independent effects on body size; however it is 
Table 4 Change in log-BMl for one SD increase in neighbourhood exposures ( $n=1813)$

\begin{tabular}{|c|c|c|c|c|c|c|}
\hline Model & Neighbourhood exposure & Coefficient & (SE) & Indirect effect & $p$-value & $\begin{array}{l}\text { Percentage of total } \\
\text { effect explained }\end{array}$ \\
\hline \multirow{5}{*}{$\begin{array}{l}1 \text { Adjusted for demographics, individual-level } \\
\text { socioeconomic factors, neighbourhood-level } \\
\text { deprivation, and neighbourhood preference }\end{array}$} & Dwelling density & -0.011036 & 0.005767 & & 0.06 & \\
\hline & Street connectivity & -0.014172 & 0.005562 & & $0.01^{*}$ & \\
\hline & Land use mix & -0.009173 & 0.005355 & & 0.09 & \\
\hline & $\mathrm{NDAl}$ & -0.012825 & 0.005395 & & $0.02^{*}$ & \\
\hline & Streetscape (SPACES) & -0.013417 & 0.004697 & & $0.007^{*}$ & \\
\hline \multirow{5}{*}{$\begin{array}{l}2 \text { Plus including physical activity (log of } \\
\text { accelerometer counts per hour) }\end{array}$} & Dwelling density & -0.009125 & 0.005768 & -0.001924 & $<0.001^{*}$ & $n / a^{a}$ \\
\hline & Street connectivity & -0.012243 & 0.005598 & -0.001889 & $<0.001^{*}$ & 13.2 \\
\hline & Land use mix & -0.008458 & 0.005297 & -0.000741 & 0.17 & 6.9 \\
\hline & $\mathrm{NDAl}$ & -0.010952 & 0.005419 & -0.00191 & $<0.001^{*}$ & 14.6 \\
\hline & Streetscape (SPACES) & -0.012626 & 0.004670 & -0.000809 & 0.11 & 5.7 \\
\hline \multirow{5}{*}{$\begin{array}{l}3 \text { Model } 1 \text { plus including percentage } \\
\text { time spent sedentary }\end{array}$} & Dwelling density & -0.0101833 & 0.0057573 & -0.000873 & 0.18 & 7.3 \\
\hline & Street connectivity & -0.0131472 & 0.0055790 & -0.001039 & 0.09 & 6.9 \\
\hline & Land use mix & -0.0089294 & 0.0053261 & -0.00025 & 0.69 & 2.4 \\
\hline & $\mathrm{NDAl}$ & -0.0118039 & 0.0054092 & -0.00109 & 0.06 & 8.1 \\
\hline & Streetscape (SPACES) & -0.0128561 & 0.0046980 & -0.000570 & 0.31 & 4.1 \\
\hline
\end{tabular}

Note: $B M I$ body mass index, $C l$ confidence interval, $n$ number, NDAI Neighbourhood Destination Accessibility Index, SD standard deviation, SPACES Systematic Pedestrian and Cycling Environment Scan

*Significant at $p<0.05$

anconsistent mediation - results not interpreted

not possible to determine which is the case from this study. With the exception of streetscape and neighbourhood-level deprivation, and mixed land use and dwelling density, significant correlations between all $\mathrm{BE}$ features were observed $(p<0.01)$, therefore collinearity was a concern.
The associations found between the BE and body size in the current study are consistent with earlier US research; when comparing the $90^{\text {th }}$ to the $10^{\text {th }}$ percentile for BE features in over 13,000 adults in New York City, Rundle et al. [59] observed reductions in BMI units of

Table 5 Change in log- waist circumference for one SD increase in neighbourhood exposures $(n=1801)$

\begin{tabular}{|c|c|c|c|c|c|c|}
\hline Model & Neighbourhood exposure & Coefficient & $(\mathrm{SE})$ & Indirect effect & $p$-value & $\begin{array}{l}\text { Percentage of total } \\
\text { effect explained }\end{array}$ \\
\hline \multirow{5}{*}{$\begin{array}{l}1 \text { Adjusted for demographics, individual-level } \\
\text { socioeconomic factors, neighbourhood-level } \\
\text { deprivation, and neighbourhood preference }\end{array}$} & Dwelling density & -0.019921 & 0.006521 & & $0.004^{*}$ & \\
\hline & Street connectivity & -0.0177428 & 0.0066846 & & $0.01^{*}$ & \\
\hline & Land use mix & -0.0020776 & 0.0067994 & & 0.76 & \\
\hline & $\mathrm{NDAl}$ & -0.0231200 & 0.0059381 & & $<0.001^{*}$ & \\
\hline & Streetscape (SPACES) & -0.0108457 & 0.0060160 & & 0.08 & \\
\hline \multirow{5}{*}{$\begin{array}{l}2 \text { Plus including physical activity (log of } \\
\text { accelerometer counts per hour) }\end{array}$} & Dwelling density & -0.017381 & 0.006388 & -0.00248 & $<0.001^{*}$ & 12.3 \\
\hline & Street connectivity & -0.0151079 & 0.0065353 & -0.00254 & $<0.001^{*}$ & 14.3 \\
\hline & Land use mix & -0.001062 & 0.006512 & -0.000962 & 0.18 & 6.6 \\
\hline & $\mathrm{NDAl}$ & -0.0206553 & 0.0058506 & -0.00242 & $<0.001^{*}$ & 10.4 \\
\hline & Streetscape (SPACES) & -0.0096708 & 0.0057933 & -0.001126 & 0.08 & 9.6 \\
\hline \multirow{5}{*}{$\begin{array}{l}3 \text { Model } 1 \text { plus including percentage time } \\
\text { spent sedentary }\end{array}$} & Dwelling density & -0.0191994 & 0.0063932 & -0.000735 & 0.23 & 3.6 \\
\hline & Street connectivity & -0.0169189 & 0.0065567 & -0.000822 & 0.17 & 4.5 \\
\hline & Land use mix & -0.0018313 & 0.0066369 & -0.000214 & 0.71 & 1.9 \\
\hline & $\mathrm{NDAl}$ & -0.0223459 & 0.0058315 & -0.000824 & 0.16 & 3.4 \\
\hline & Streetscape (SPACES) & -0.0102434 & 0.0058882 & -0.000600 & 0.27 & 5.0 \\
\hline
\end{tabular}

Note: $B M I$ body mass index, $n$ number, $C l$ confidence interval, NDAI Neighbourhood Destination Accessibility Index, SD standard deviation, SPACES Systematic Pedestrian and Cycling Environment Scan

*Significant at $p<0.05$ 
$0.41,0.33,0.34$, and $2.86 \mathrm{~kg} / \mathrm{m}^{2}$ with increases in mixed land use, bus stop density, subway stop density, and population density, respectively. Likewise, in a study of over 16,000 Texas adults, Hoehner et al. [30] determined that men and women living in neighbourhoods $1 \mathrm{SD}$ above the mean for older homes (proxy measure of more walkable neighbourhoods) and shorter commute times had BMI values of 0.77 and $0.84 \mathrm{~kg} / \mathrm{m} 2$ lower than those living in neighbourhoods $1 \mathrm{SD}$ below the mean.

Advancing on this earlier work, the mediating effects of objectively assessed physical activity and sedentary behaviour on this relationship were considered. Consistent with earlier research $[28,32]$, no mediating effect was found for sedentary behaviour. While clear associations exist between sedentary time (particularly prolonged sitting) and body size in adults [60, 61], studies of associations between the $\mathrm{BE}$ and sedentary time are scarce [62]. It may be hypothesised from this small but consistent evidence base that a substantial relationship between the $\mathrm{BE}$ and objectively assessed sedentary time is unlikely. Conversely, it is also plausible that a clear understanding of this relationship has been limited by methodological issues, and lack of sensitivity and specificity in quantifying outcomes, particularly sedentary behaviours. For example, while improving on selfreported behaviours, the use of accelerometry to measure time spent sedentary is still imprecise; postural transitions cannot be assessed, and consensus is lacking on best practice for measurement protocols, data processing, cleaning, and aggregation [63]. We recommend employing additional methods in future to explore possible relationships between sedentary time and the $\mathrm{BE}$ in more detail (e.g., inclinometers, life-logging cameras, global positioning systems combined with accelerometry).

In contrast, a significant mediating effect of physical activity was observed on the relationship between body size and NDAI and street connectivity. These findings are consistent with the limited research that has considered the mediating effects of physical activity on the relationship between the BE and BMI [28-32, 64], and confirm the hypothesis that the relationship between $\mathrm{BE}$ and body size, mediated by physical activity, is not the result of systematic error. Preliminary analyses (not reported here) showed that the addition of neighbourhood preference to modelling resulted in no changes to relationships and only minor changes in the magnitude of differences. It is worth noting that when selecting their preferred neighbourhood, participants were requested to assume uniformity across neighbourhood types with respect to key issues such as schools, neighbourhood demographics and housing cost, which may have confounded findings somewhat as it is an idealistic measure. In addition, the neighbourhood preference measure employed was relatively broad, offering participants a choice of two neighbourhoods, essentially dichotomised by features that enable or disable use of active and public transport modes; consequently, selection effects may not have been captured completely.

The current study improves on limitations in previous research in this field [7, 65] by: undertaking robust assessment of the BE using contemporary GIS databases; purposefully selecting heterogeneous neighbourhoods in terms of walkability and region; randomly selecting participants; objectively assessing physical activity and body size (using BMI and waist circumference); using a continuous measure of BMI rather than thresholds (which can bias results [66]; a consideration especially important given the ethnic diversity in this study); adjusting for neighbourhood preference; and utilising mediation analyses to consider the effect of physical activity on the BE-body size relationship. Debate regarding approaches for accelerometer data reduction approach is ongoing [67], and to date, no agreed-upon best-practice method exists. In this study we employed a pragmatic accelerometer data reduction protocol that enabled inclusion of data for all complete hours of wear time (as opposed to limits of hours/days of wear), with the aim of reducing bias (for example with less compliant participants, [55]). Mean accelerometer counts were used to describe physical activity, recognising the issues surrounding the use of accelerometer count thresholds, and the contribution of activity of any intensity to health [68].

We did not estimate associations with individual $\mathrm{BE}$ characteristics with adjustments for all other $\mathrm{BE}$ features in the same model (due to collinearity) - this means that associations with each $\mathrm{BE}$ feature are not independent of associations with other BE attributes (as they are likely to be confounded by these variables). Caution should be applied when generalising these findings internationally, considering the substantial variability in urban form across countries [69]. It is also worth noting we did not consider the food environment in this study. In their earlier research, Brown et al. [29] undertook detailed and robust assessment of average caloric intake (two 24-h recalls); when controlling for this factor, a significant mediating effect of objectively-assessed physical activity still remained for the relationship between the $\mathrm{BE}$ and BMI. It is possible that clustering or collinearity exists between $\mathrm{BE}$ features that support physical activity and healthy nutritional practices; it is also plausible that the food environment (e.g., density of fast food outlets) may play an additive role with the physical activity environment in predicting body size.

While this study contributes to the cross-sectional evidence base, it does not enable the identification of causal relationships. Moreover, mediation analyses with crosssectional data (particularly when stratified such as in the 
current study) can be subject to collider bias and measurement error [70]. Study findings are limited to New Zealand adults only; it is possible that differential findings may be observed for younger and older population groups, and for other population groups. With a response rate of $44 \%$, it is possible that self selection bias was present in the current study sample, although it is worth noting this response rate is higher than similar studies (e.g., $12 \%$ in the study of Owen et al. [71], and $26 \%$ in Frank et al. [37]).

Examination of relationships between objectively assessed body size, nutrition behaviours, physical activity, and food environments in future population research (particularly including younger and older participants) would be challenging, but an important next step in determining the relative contributions of behaviours and environmental features to obesity risk for people of all ages. Longitudinal, repeated measurements of $\mathrm{BE}$ exposures, risk behaviours, and body size outcomes are also necessary. Making use of existing longitudinal health datasets and alignign BE measurement with routine population studies are approaches that may make this feasible.

\section{Conclusion}

This research contributes to the growing evidence base for links between the built environment and body size, and for the mediating effect of physical activity on this relationship. Longitudinal research, including objective outcome and environmental measures, across a range of demographically and geographically diverse population groups is needed to determine causality and generalisability of these relationships.

\section{Abbreviations \\ BMI: Body Mass Index; BRFSS: Behavioral risk factor surveillance system; GIS: Geographic information systems; IPEN: International Physical Activity and Environment Network; MB: Meshblock; NDAl: Neighbourhood Destinations Accessibility Index; NZ: New Zealand; SPACES: Systematic Pedestrian and Cycling Environment Scan; SD: standard deviation; WC: Waist circumference; URBAN: Understanding the Relationship between Activity and Neighbourhoods study.}

\section{Competing interests}

The authors declare that they have no competing interests.

\section{Authors' contributions}

$H M B, G S, K W, R A K, S M$, and EH originally conceived of and designed the URBAN study; MO, TB, and VI further contributed to the study design and coordination. HMB led the data collection and data cleaning. MO, KW, TB, and GS conceptualised and led the current investigation. KP, TB, and PS conceptualised and undertook data treatment and analyses. SM led the built environment measurement. JP and RAK provided geographic expertise and input to the mansucript draft; JP led the development and construction of the NDAI. MO drafted the manuscript. All authors read, contributed to, and approved the manuscript.

\section{Acknowledgements}

The URBAN Study and the Neighbourhood and Health Study were funded by the Health Research Council of New Zealand. We thank all members of the URBAN team as well as the study participants for their contribution to the research. The authors are independent from the funder and the funder had no role in the design and conduct of the study, or the preparation of the manuscript.

\section{Authors' information}

Not applicable.

\section{Availability of data and materials}

Not applicable.

\section{Funding}

We thank all members of the Understanding the Relationship between Activity and Neighbourhoods (URBAN) team, as well as the study participants, for their contributions to the research. The URBAN Study was funded by the Health Research Council of New Zealand. The authors are independent of the funder, and the funder had no role in the design and conduct of the study or in the preparation of the manuscript. The authors declare they have no actual or potential competing financial interests.

\section{Author details}

'Human Potential Centre, Auckland University of Technology, Auckland, New Zealand. ${ }^{2}$ SHORE and Whāriki Research Centre, Massey University, Auckland, New Zealand. ${ }^{3}$ Department of Public Health, University of Otago, Wellington, New Zealand. ${ }^{4}$ McCaughey VicHealth Community Wellbeing Unit, The University of Melbourne, Melbourne, Australia. ${ }^{5}$ School of GeoSciences, University of Edinburgh, Edinburgh, Scotland, UK. ${ }^{6}$ School of Environment, The University of Auckland, Auckland, New Zealand.

Received: 15 April 2015 Accepted: 16 September 2015

Published online: 24 September 2015

\section{References}

1. Nguyen DM, El-Serag HB. The epidemiology of obesity. Gastroenterol Clin North Am. 2010;39(1):1-7. doi:10.1016/j.gtc.2009.12.014.

2. Mackenbach JD, Rutter H, Compernolle S, Glonti K, Oppert J-M, Charreire H, et al. Obesogenic environments: A systematic review of the association between the physical environment and adult weight status, the SPOTLIGHT project. BMC Public Health. 2014:14:233. doi:10.1186/1471-2458-14-233.

3. World Health Organization. Obesity: preventing and managing the global epidemic: report of a WHO consultation. Geneva, Switzerland: Author;2004.

4. Papas MA, Alberg AJ, Ewing R, Helzlsouer KJ, Gary TL, Klassen AC. The built environment and obesity. Epidemiol Rev. 2007 doi:10.1093/epirev/mxm009.

5. Lake AA, Townshend TG, Alvanides S. Obesogenic environments. Complexities, perceptions, and objective measures. Sussex, UK: Wiley-Blackwell; 2010.

6. Booth KM, Pinkston MM, Poston WS. Obesity and the built environment. J Am Diet Assoc. 2005;105(5 Suppl 1):S110-7. doi:10.1016/j.jada.2005.02.045.

7. Ding D, Gebel K. Built environment, physical activity, and obesity: what have we learned from reviewing the literature? Health Place. 2012;18(1):100-5. doi:10.1016/j.healthplace.2011.08.021.

8. Sallis JF, Floyd MF, Rodriguez DA, Saelens BE. Role of built environments in physical activity, obesity, and cardiovascular disease. Circulation. 2012;125(5):729-37. doi:10.1161/CIRCULATIONAHA.110.969022

9. Sallis JF, Saelens BE, Frank LD, Conway TL, Slymen DJ, Cain KL, et al. Neighborhood built environment and income: examining multiple health outcomes. Soc Sci Med. 2009;68(7):1285-93. doi:10.1016/j.socscimed. 2009.01.017.

10. Feng J, Glass TA, Curriero FC, Stewart WF, Schwartz BS. The built environment and obesity: a systematic review of the epidemiologic evidence. Health Place. 2010;16:175-90.

11. Sugiyama T, Koohsari MJ, Mavoa S, Owen N. Activity-friendly built environment attributes and adult adiposity. Curr Obes Rep. in press.

12. Stafford M, Cummins S, Ellaway A, Sacker A, Wiggins RD, Macintyre S. Pathways to obesity: identifying local, modifiable determinants of physical activity and diet. Soc Sci Med. 2007;65(9):1882-97. doi:10.1016/j.socscimed.2007.05.042.

13. Frank LD, Saelens BE, Powell KE, Chapman JE. Stepping towards causation: do built environments or neighborhood and travel preferences explain physical activity, driving, and obesity? Soc Sci Med. 2007;65:1898-914. doi:10.1016/j.socscimed.2007.05.053.

14. Black JL, Macinko J. Neighborhoods and obesity. Nutr Rev. 2008;66(1):2-20. doi:10.1111/j.1753-4887.2007.00001.x.

15. Lebel A, Kestens Y, Pampalon R, Theriault M, Daniel M, Subramanian SV. Local context influence, activity space, and foodscape exposure in two 
canadian metropolitan settings: Is daily mobility exposure associated with overweight? J Obes. 2012;2012:912645. doi:10.1155/2012/912645.

16. Committee on Physical Activity $H$, Transportation, and Land Use. Does the built environment influence physical activity? Washington, DC: The National Academy of Sciences; 2005.

17. Transportation Research Board and Institute of Medicine of the National Academies. Does the built environment influence physical activity? Examining the evidence. Washington, DC: Transportation Research Board, Institute of Medicine of the National Academies; 2005.

18. Witten K, Blakely T, Bagheri N, Badland H, Ivory V, Pearce J, et al. Neighborhood built environment and transport and leisure physical activity: Findings using objective exposure and outcome measures in New Zealand. Environ Health Perspect. 2012;120(7):971-7. doi:10.1289/ehp.1104584.

19. Christian H, Giles-Corti B, Knuiman M, Timperio A, Foster S. The influence of the built environment, social environment and health behaviors on body mass index. results from RESIDE. Prev Med. 2011;53(1-2):57-60. doi:10.1016/ j.ypmed.2011.05.004.

20. Badland HM, Oliver M, Kearns RA, Mavoa S, Witten K, Duncan MJ, et al. Association of neighbourhood residence and preferences with the built environment, work-related travel behaviours, and health implications for employed adults: Findings from the URBAN study. Soc Sci Med. 2012;75(8):1469-76.

21. Berry TR, Spence JC, Blanchard CM, Cutumisu N, Edwards J, Selfridge G. A longitudinal and cross-sectional examination of the relationship between reasons for choosing a neighbourhood, physical activity and body mass index. Int J Behav Nutr Phys Act. 2010;7:57. doi:10.1186/1479-5868-7-57.

22. Cao XY, Mokhtarian PL, Handy SL. Examining the impacts of residential self-selection on travel behaviour: a focus on empirical findings. Transport Rev. 2009;29(3):359-95. doi:10.1080/01441640802539195.

23. Frank LD, Sallis JF, Conway TL, Chapman JE, Saelens BE, Bachman W. Many pathways from land use to health - Associations between neighborhood walkability and active transportation, body mass index, and air quality. J Am Plann Assoc. 2006;72(1):75-87. doi:10.1080/01944360608976725.

24. Zick CD, Smith KR, Fan JX, Brown BB, Yamada I, Kowaleski-Jones L. Running to the store? The relationship between neighborhood environments and the risk of obesity. Soc Sci Med. 2009;69(10):1493-500. doi:10.1016/ j.socscimed.2009.08.032.

25. Connor Gorber S, Tremblay M, Moher D, Gorber B. A comparison of direct vs. self-report measures for assessing height, weight and body mass index: a systematic review. Obes Rev. 2007;8(4):307-26. doi:10.1111/j.1467789X.2007.00347.X

26. Wilcox S, King AC. Self-favoring bias for physical activity in middle-aged adults. J Appl Soc Psychol. 2000;30(9):1773-89.

27. Menke A, Muntner P, Wildman RP, Reynolds K, He J. Measures of adiposity and cardiovascular disease risk factors. Obesity. 2007;15:785-95.

28. Van Dyck D, Cerin E, Cardon G, Deforche B, Sallis JF, Owen N, et al. Physical activity as a mediator of the associations between neighborhood walkability and adiposity in Belgian adults. Health Place. 2010;16(5):952-60. doi:10.1016/ j.healthplace.2010.05.011.

29. Brown BB, Smith KR, Hanson H, Fan JX, Kowaleski-Jones L, Zick CD. Neighborhood design for walking and biking. Physical activity and body mass index. Am J Prev Med. 2013:44(3):231-8. doi:10.1016/j.amepre. 2012.10.024

30. Hoehner CM, Handy SL, Yan Y, Blair SN, Berrigan D. Association between neighborhood walkability, cardiorespiratory fitness and body-mass index. Soc Sci Med. 2011;73(12):1707-16. doi:10.1016/j.socscimed.2011.09.032.

31. Siceloff ER, Coulon SM, Wilson DK. Physical activity as a mediator linking neighborhood environmental supports and obesity in African Americans in the PATH Trial. Health Psychol. 2014;33(5):481-9. doi:10.1037/a0032758

32. Oyeyemi AL, Deforche B, Sallis JF, De Bourdeaudhuij I, Van Dyck D. Behavioral mediators of the association between neighborhood environment and weight status in Nigerian adults. Am J Health Promot. 2013;28(1):23-31. doi:10.4278/ajhp.120509-QUAN-244.

33. Hamilton MT, Hamilton DG, Zderic TW. Role of low energy expenditure and sitting in obesity, metabolic syndrome, type 2 diabetes, and cardiovascular disease. Diabetes. 2007;56(11):2655-67.

34. Van Dyck D, Cerin E, Conway TL, De Bourdeaudhuij I, Owen N, Kerr J, et al. Associations between perceived neighborhood environmental attributes and adults' sedentary behavior: Findings from the USA, Australia and Belgium. Soc Sci Med. 2012;74(9):1375-84.
35. Badland HM, Schofield GM, Witten K, Schluter PJ, Mavoa S, Kearns RA, et al. Understanding the Relationship between Activity and Neighbourhoods (URBAN) study: Research design and methodology. BMC Public Health. 2009;9:224.

36. IPEN. Home page [International Physical Activity and the Environment Network]. 2001. http://www.ipenproject.org/. Accessed August 222013.

37. Frank LD, Sallis JF, Saelens BE, Leary L, Cain K, Conway TL, et al. The development of a walkability index: application to the neighborhood quality of life study. Br J Sports Med. 2010;44:924-33. doi:10.1136/bjsm.2009.058701.

38. Statistics New Zealand. 2006 Census of Population and Dwellings. Wellington, New Zealand: Author;2007.

39. Statistics New Zealand. New Zealand in profile: An overview of New Zealand's people, economy, and environment. Wellington, New Zealand: Author;2009.

40. Marfell-Jones M, Olds T, Stewart A, Carter L. International Standards for Anthropometric Assessment. Potchefstroom, South Africa: International Society for the Advancement of Kinanthropometry (ISAK). 2006.

41. Leslie E, Coffee N, Frank L, Owen N, Bauman A, Hugo G. Walkability of local communities: using geographic information systems to objectively assess relevant environmental attributes. Health Place. 2007;13(1):111-22. doi:10.1016/j.healthplace.2005.11.001.

42. Witten K, Pearce J, Day P. Neighbourhood Destination Accessibility Index: A GIS tool for measuring infrastructure support for neighbourhood physical activity. Environ Plann A. 2011:43(1):205-23.

43. Salmond C, Crampton P, Atkinson J. NZDep2006 Index of Deprivation. Wellington, NZ: Department of Public Health, University of Otago; 2007.

44. Pikora T, Bull FCL, Jamrozik K, Knuiman M, Giles-Corti B, Donovan RJ. Developing a reliable audit instrument to measure the physical environment for physical activity. Am J Prev Med. 2002;23(3):187-94.

45. Badland HM, Opit S, Witten K, Kearns RA, Mavoa S. Can virtual streetscape audits reliably replace physical streetscape audits? J Urban Health. 2010;87(6):1007-16. doi:10.1007/s11524-010-9505-x.

46. Oliver M, Badland HM, Schofield GM, Shepherd J. Identification of accelerometer non-wear time and sedentary behavior. Res Q Exerc Sport. 2011;82(4):593-7

47. Wong SL, Colley R, Gorber SC, Tremblay M. Actical accelerometer sedentary activity thresholds for adults. J Phys Act Health. 2011:8:587-91.

48. Ministry of Social Development. Household incomes in New Zealand: Trends in indicators of inequality and hardship 1982 to 2010. Wellington, New Zealand: Author;2011.

49. Levine J, Inam A, Torng G-W. A choice-based rationale for land use and transportation alternatives: evidence from Boston and Atlanta. J Plann Educ Res. 2005;24(3):317-30.

50. Owen N, Bauman A, Brown W. Too much sitting: a novel and important predictor of chronic disease risk? Br J Sports Med. 2009;43:81-3.

51. Hamilton MT, Healy GN, Dunstan DW, Zderic TW, Owen N. Too little exercise and too much sitting: inactivity physiology and the need for new recommendations on sedentary behavior. Curr Cardiovasc Risk Rep. 2008;2(4):292-8. doi:10.1007/s12170-008-0054-8.

52. Karndacharuk A, Wilson DJ, Dunn RCM. Analysis of pedestrian performance in shared space environments. Transp Res Rec. 2013;2393:1-11.

53. Feng Z, Raghuwanshi RP, Xu Z. Electric-bicycle-related injury: a rising traffic injury burden in China. Inj Prev. 2010;16(6):417-9.

54. Tingley D, Yamamoto T, Hirose K, Keele L, Imai K. mediation: R package for causal mediation analysis. J Stat Softw. 2014;59:5.

55. Toftager M, Kristensen PL, Oliver M, Duncan S, Christiansen LB, Boyle E, et al. Accelerometer data reduction in adolescents: effects on sample retention and bias. Int J Behav Nutr Phys Act. 2013;10:140. doi:10.1186/ 1479-5868-10-140.

56. New Zealand Transport Agency. Travel Planning Toolkit Guidelines and Resources. Resource 1. Facts and Figures. Wellington, New Zealand: Author;2011.

57. Key Labour Force Measures by Qualification, Age and Sex (2008) [database on the Internet]. Author. 2008. Available from: http://nzdotstat.stats.govt.nz/ wbos/Index.aspx?DataSetCode=TABLECODE7080. Accessed: 14 March, 2014

58. MacKinnon DP, Fairchild AJ, Fritz MS. Mediation analysis. Annu Rev Psychol. 2007:58:593-614. doi:10.1146/annurev.psych.58.110405.085542.

59. Rundle A, Diez Roux AV, Free LM, Miller D, Neckerman KM, Weiss CC. The urban built environment and obesity in New York City: a multilevel analysis. Am J Health Promot. 2007;21(4 Suppl):326-34.

60. Healy GN, Dunstan DW, Salmon J, Cerin E, Hawley JA, Owen N. Prolonged bouts of sedentary time are associated with percent body-fat, independent 
of total sedentary time. Med Sci Sports Exerc. 2007;35(5 Suppl):S12. doi:10.1249/01.mss.0000272920.61887.40.

61. Healy GN, Wijndaele K, Dunstan DW, Shaw JE, Salmon J, Zimmet PZ, et al. Objectively measured sedentary time, physical activity, and metabolic risk: the Australian Diabetes, Obesity and Lifestyle Study (AusDiab). Diabetes Care. 2008;31(2):369-71.

62. Sugiyama T, Salmon J, Dunstan DW, Bauman AE, Owen N. Neighborhood walkability and TV viewing time among Australian adults. Am J Prev Med. 2007;33:444-9.

63. Atkin AJ, Gorely T, Clemes SA, Yates T, Edwardson C, Brage S, et al. Methods of measurement in epidemiology: sedentary behaviour. Int J Epidemiol. 2012;41(5):1460-71. doi:10.1093/ije/dys118.

64. Richardson EA, Pearce J, Mitchell R, Kingham S. Role of physical activity in the relationship between urban green space and health. Public Health. 2013;127(4):318-24. doi:http://dx.doi.org/10.1016/.ppuhe.2013.01.004.

65. Handy S, Cao XY, Mokhtarian PL. Self-selection in the relationship between the built environment and walking - Empirical evidence from northern California. J Am Plann Assoc. 2006;72(1):55-74. doi:10.1080/ 01944360608976724.

66. World Health Organization. The Asia-Pacific perspective: Redefining obesity and its treatment. Geneva: Author;2000.

67. Mâsse LC, Fuemmeler BF, Anderson CB, Matthews CE, Trost SG, Catellier DJ, et al. Accelerometer data reduction: A comparison of four reduction algorithms on select outcome variables. Med Sci Sports Exerc. 2005;37(11 Suppl):S544-S54

68. Levine JA, Vander Weg MW, Hill JO, Klesges RC. Non-exercise activity thermogenesis: The crouching tiger hidden dragon of societal weight gain Arterioscler Thromb Vasc Biol. 2006;26(4):729-36.

69. Goodman A, Sahlqvist S, Ogilvie D. New walking and cycling routes and increased physical activity: One- and 2-year findings from the UK iConnect Study. American Journal of Public Health. 2014:e1-e9. doi:10.2105/ AJPH.2014.302059.

70. Cole SR, Platt RW, Schisterman EF, Chu HT, Westreich D, Richardson D, et al. Illustrating bias due to conditioning on a collider. Int J Epidemiol. 2010;39(2):417-20. doi:10.1093/lje/Dyp334.

71. Haase JE, Heiney SP, Ruccione KS, Stutzer C. Research triangulation to derive meaning-based quality-of-life theory: adolescent resilience model and instrument development. Int J Cancer Suppl. 1999;12:125-31. doi:10.1002/ (SICI) 1097-0215(1999)83:12+<125:.AID-IJC22>3.0.CO;2-7.

\section{Submit your next manuscript to BioMed Central and take full advantage of:}

- Convenient online submission

- Thorough peer review

- No space constraints or color figure charges

- Immediate publication on acceptance

- Inclusion in PubMed, CAS, Scopus and Google Scholar

- Research which is freely available for redistribution 